\title{
Combined effect of interventions with pure or enriched mixtures of (poly)phenols and anti-diabetic medication in type 2 diabetes management: a meta-analysis of randomized controlled human trials
}

\author{
Ana F. Raimundo ${ }^{1,2,3} \cdot$ Filipa Félix $^{1,3} \cdot$ Rita Andrade $^{4} \cdot$ María-Teresa García-Conesa $^{5} \cdot$ Antonio González-Sarrías $^{5}$. \\ João Gilsa-Lopes ${ }^{2} \cdot{\text { Dulce do } \mathrm{O}^{4} \cdot \text { Ana Raimundo }}^{4} \cdot$ Rogério Ribeiro $^{2,4,6} \cdot$ Ana Rodriguez-Mateos $^{7}$. \\ Cláudia N. Santos ${ }^{1,2,3} \cdot$ Manuel Schär $^{8} \cdot$ Ana Silva ${ }^{9} \cdot$ Inês Cruz ${ }^{9} \cdot$ Brian Wang $^{7} \cdot$ Paula Pinto $^{3,9,10} \cdot$ Regina Menezes $^{1,2,3}$ (1)
}

Received: 2 August 2019 / Accepted: 24 January 2020 / Published online: 12 February 2020

(c) Springer-Verlag GmbH Germany, part of Springer Nature 2020

\begin{abstract}
Purpose (Poly)phenols have been reported to confer protective effects against type 2 diabetes but the precise association remains elusive. This meta-analysis aimed to assess the effects of (poly)phenol intake on well-established biomarkers in people with type 2 diabetes or at risk of developing diabetes.

Methods A systematic search was conducted using the following selection criteria: (1) human randomized controlled trials involving individuals with prediabetes and type 2 diabetes; (2) one or more of the following biomarkers: glucose, glycated haemoglobin (HbA1c), insulin, pro-insulin, homeostatic model assessment of insulin resistance (HOMA-IR), islet amyloid polypeptide (IAPP)/amylin, pro-IAPP/pro-amylin, glucagon, C-peptide; (3) chronic intervention with pure or enriched mixtures of (poly)phenols. From 488 references, 88 were assessed for eligibility; data were extracted from 27 studies and 20 were used for meta-analysis. The groups included in the meta-analysis were: (poly)phenol mixtures, isoflavones, flavanols, anthocyanins and resveratrol.

Results Estimated intervention/control mean differences evidenced that, overall, the consumption of (poly)phenols contributed to reduced fasting glucose levels $(-3.32 \mathrm{mg} / \mathrm{dL} ; 95 \% \mathrm{CI}-5.86,-0.77 ; P=0.011)$. Hb1 Ac was only slightly reduced $(-0.24 \% ; 95 \% \mathrm{CI}-0.43,-0.044 ; P=0.016)$ whereas the levels of insulin and HOMA-IR were not altered. Subgroup comparative analyses indicated a stronger effect on blood glucose in individuals with diabetes $(-5.86 \mathrm{mg} / \mathrm{dL}, 95 \% \mathrm{CI}-11.34$, $-0.39 ; P=0.036)$ and this effect was even stronger in individuals taking anti-diabetic medication $(-10.17 \mathrm{mg} / \mathrm{dL}, 95 \% \mathrm{CI}$ $-16.59,-3.75 ; P=0.002)$.

Conclusions Our results support that the consumption of (poly)phenols may contribute to lower glucose levels in individuals with type 2 diabetes or at risk of diabetes and that these compounds may also act in combination with anti-diabetic drugs.
\end{abstract}

Keywords Antidiabetic therapy $\cdot$ Diabetes $\cdot$ Glucose $\cdot \mathrm{Hb} 1 \mathrm{Ac} \cdot$ Insulin $\cdot$ Polyphenol

\section{Introduction}

Type 2 diabetes mellitus (hereafter referred as diabetes) affects hundreds of millions of people worldwide, being projected that by 2040 the number of individuals with diabetes will reach 642 million [1]. The International Diabetes

Paula Pinto

paula.pinto@esa.ipsantarem.pt

Regina Menezes

rmenezes@ibet.pt; regina.menezes@nms.unl.pt

Extended author information available on the last page of the article
Federation estimated that $12 \%$ of global health expenditure is spent on diabetes [1] and, despite the advances in both modern medicine and knowledge of the disease, it remains one of the leading causes of death globally.

It is generally considered that diabetes results from the combination of genetic and environmental factors. Different loci have been put forward as risk factors for diabetes [2], however, lifestyle factors such as obesity, lack of physical exercise, calorie-rich diets, and smoking have been considered the greatest players in the disease [3]. These are potentially modifiable factors which can delay the disease onset and progression. Together with proper monitoring 
and medication, lifestyle and dietary changes are essential to control diabetes and avoid its comorbidities [4].

Glucose homeostasis results from a controlled system in which the rise of blood glucose is compensated by the increase of insulin secretion. In healthy states, this tightly regulated feedback loop guarantees that glucose levels remain within the normal range. Insulin resistance and impaired insulin secretion are the major pathological processes associated with diabetes development. Insulin resistance precedes diabetes onset and occurs when cells fail to respond to physiological levels of insulin, mainly in the liver and in the muscles [5]. Impaired insulin secretion is characterized by the lack of proper secretion of insulin by the $\beta$-cells in response to glucose as a result of decreased $\beta$-cell function and mass $[6,7]$. This can also lead to the release of an immature form of insulin, before C-peptide cleavage pro-insulin $[8,9]$. These mechanisms culminate in hyperglycaemia, the major clinical symptom of diabetes. Another established hallmark of diabetes, yet clinically unexplored, is the formation of pancreatic deposits of islet amyloid polypeptide (IAPP) or amylin [10], which are associated with $\beta$-cell failure. IAPP is co-secreted with insulin by pancreatic $\beta$-cells and plays a role in glucose homeostasis by regulating satiety and gastric emptying [8]. Failure of $\beta$-cell processing machinery during diabetes is also associated with secretion of pro-IAPP forms [11, 12]. Locally subjected to high levels of insulin, $\alpha$-cells release increased levels of glucagon, thus hyperglucagonemia is also commonly found in diabetes [13].

(Poly)phenols comprise a large class of phytochemicals present in diverse sources in the diet, such as fruit, vegetables, red wine, and cocoa [14]. They are known to exhibit a variety of biological activities targeting different molecular mechanisms and cellular pathways [15]. The beneficial health effects of (poly)phenols have been associated with protection against cardiovascular diseases (e.g., promoting endothelial function and inhibiting platelet aggregation), cancer (via the reduction of cell proliferation, induction of cell cycle arrest or apoptosis) [16], neurological disorders such as Parkinson's and Alzheimer's diseases and diabetes (e.g., modulating oxidative stress and anti-inflammatory responses) $[17,18]$. Particularly, human studies have shown the correlation between (poly)phenol-rich food consumption and reduced risk of developing diabetes $[19,20]$. For example, the prospective examination of the associations between (poly)phenols intake on the risk of incident diabetes in the PREDIMED study revealed a $28 \%$ reduction in new-onset diabetes in the highest compared with the lowest tertile of total (poly)phenol intake (Hazard Ratio-HR 0.72; 95\% CI $0.52,0.99 ; P$-trend $=0.05$ ). Notably, a high intake of (poly) phenols was inversely associated with diabetes in elderly persons at high risk of cardiovascular disease [21]. Also, dietary (poly)phenols were inversely associated with metabolic syndrome in adults of the HAPIEE study, as individuals in the highest quartile of (poly)phenol intake were less likely to develop the syndrome (Odds Ratio-OR 0.80; 95\% CI $0.64,0.98$ and OR $0.70 ; 95 \%$ CI $0.56,0.86$ for both men and women, respectively) [22]. Additionally, isoflavone-rich soybased foods were reported to reduce significantly the risk of diabetes (OR for the highest versus the lowest intake: 0.31 ; 95\% CI 0.21, 0.46; $P<0.001$ ) [23]. (Poly)phenols are also thought to contribute to the control of this disease [24] as exemplified by flavonoid-rich berries and oolong tea consumption that improved post-prandial hyperglycaemia [25, 26]. Animal studies further support the beneficial effects of (poly)phenol-enriched mixtures and pure extracts intake against altered glucose metabolism. A study using the flavonoid kaempferol reported improved insulin resistance in a diabetes rat model potentially through the reduction of hepatic inflammation by the inhibition of the NF- $\mathrm{KB}$ pathway [27]. Another report showed a significant reduction of hyperglycaemia and insulin resistance in diabetes rats as well as a modulation of inflammation following treatment with ellagic acid [28]. Different (poly)phenols such as resveratrol and the ellagitannin pentagalloyl-glucose have also been described to prevent the aggregation of IAPP and to reduce its cellular toxicity using in vitro models $[29,30]$.

Overall, (poly)phenols have been widely substantiated by pre-clinical evidence as compounds with metabolic regulatory effects that may contribute to prevent or delay the onset of type- 2 diabetes but the evidences in humans are still limited. There are only a few meta-analyses of randomized controlled human trials evaluating the effects of (poly)phenols on diabetes biomarkers or incidence, and some of those report ambiguous results [31-36]. For instance, effects on biomarkers such as blood glucose, fasting insulin or $\mathrm{HbA} 1 \mathrm{c}$ are reported to be changed by a certain (poly)phenol in one meta-analysis and not altered in another [32, 33]. In addition, and despite that most individuals with diabetes are under medication, very few of these studies have contemplated a possible combined effect between (poly)phenols and anti-diabetic drugs. Overall, the protective role of dietary (poly)phenols towards diabetes is not yet understood. In the present study, we systematically reviewed published randomized controlled trials investigating the impact of (poly)phenol consumption on diabetes biomarkers. Our main aim was to analyse the reported effects on a selection of biomarkers associated with the disorder, i.e., blood glucose, glycated haemoglobin (HbA1c), insulin, pro-insulin, insulin resistance (HOMA-IR), IAPP/amylin, pro-IAPP/pro-amylin, glucagon and C-peptide. The influence of factors that may be responsible for inter-individual variability in the response to (poly)phenol consumption was also examined, including sex, BMI, health status and prescribed medication. The influence of type of (poly)phenol, dose and duration of treatment was also investigated. 


\section{Methods}

\section{Search strategy, study selection and data extraction}

This systematic review and meta-analysis followed the Preferred Reporting Items for Systematic Reviews and Meta-Analyses (PRISMA) statement guidelines [37], and the Cochrane Handbook for Systematic Reviews of Interventions [38]. A systematic search was conducted by two of the authors in Medline, SCOPUS, Web of Knowledge and ClinicalTrials.gov in November 2016 and updated on January 2018, to select randomized clinical trials that studied the effects of pure (poly)phenols on diabetes.

Search terms in titles and abstracts included (the wild card "*" was used to increase the number of matches): polyphenol* OR flavonoid* OR anthocyani* OR flavanol OR flavonol OR flavone OR flavanone OR ellagitannin OR proanthocyani* OR "phenolic acid" OR resveratrol, AND diabet* OR prediabet* NOT rat AND clinical trial (MeSH terms were used in PubMed). The search was restricted to English language.

After removal of duplicates, studies were screened by two independent authors, and double-checked by a third author to reach consensus of selected studies for full paper eligibility. Selected studies were limited to human randomized controlled trials, which (1) had a chronic intervention (4 weeks or more), with a pure (poly)phenol or an enriched fraction of (poly)phenols and a control group receiving a placebo and (2) measured one or more of the following outcomes: blood glucose, HbA1c, insulin, proinsulin, HOMA-IR, IAPP/amylin, pro-IAPP/pro-amylin, glucagon and C-peptide.

Data extraction was performed independently by two authors using a standardized data extraction form and crosschecked by a third author. Extracted data included: (1) publication details: name of first author, year of publication, title, name and e-mail of corresponding author; (2) study characteristics: study design, arm number and description, washout duration, treatment duration, number of participants under (poly)phenol supplementation, number of participants receiving the placebo, number of participants completing the study, composition of (poly) phenol supplement and placebo, dose and mode of administration; (3) sample characteristics: country, number of male and female participants, age mean and age range, ethnicity, health status (diabetes, moderate risk-overweight, obesity, first-degree relatives with diabetes or peripheral insulin resistance-and high risk-metabolic syndrome or impaired glucose), menopausal status, smoking, medication, baseline BMI, diet (assessment method, baseline diet and diet during study), physical activity level; (4) information on reported outcomes: type of sample (fasted or post-prandial), changes or values before and after intervention (central measure, dispersion measure and $P$ value when available); (5) Outcomes: glucose, HbAlc, insulin, pro-insulin, HOMA-IR, IAPP/amylin, pro-IAPP/proamylin, glucagon, and C-peptide. Before analysis, glucose and insulin units were converted to the most commonly used units in the clinical environment $(\mathrm{mg} / \mathrm{dL}$ for glucose and $\mu \mathrm{IU} / \mathrm{mL}$ for insulin).

\section{Assessment of bias}

A systematic assessment of the risk of bias for each of the included studies was based on the Cochrane Collaboration measurement with some modifications [38]. The specific items used for the assessments of each study are those used in a previous meta-analysis [39]: (1) selection bias-random sequence generation, allocation concealment (in each item, yes $=1$; no $=0$, unclear $=0$ ); (2) performance bias-blinding (yes $=1$ for each participants, researchers and statisticians, no $=0$, unclear $=0)$, measurement of compliance ( 1 for biomarker measure, 0.5 if compliance information was collected by counting non-used capsules or recipients, or by self-reporting, 0 if no measurement of compliance was done or the information was insufficient); (3) attrition bias-flow of participants ( 1 if flow of participants was explained in detail, including number of withdrawals and reasons, 0 if there was no information or insufficient information); (4) other bias-baseline comparability between test and control groups (yes $=1$, no $=0$, unclear $=0$ ), data report ( 1 if preand post-data or change was reported in a table with central measure and spread for placebo and treatment groups, and sample size in each group, 0 if anything was missing), industry funding ( 0 if any commercial source provided some or all monetary funding for the trial, if a company carried out a study "in house", if any of the authors was employed by a relevant industry or if it was unclear that there was any kind of industry funding, 1 if there was no funding from industry or if the only involvement of a company was to provide some ingredients for the intervention). Studies were rated as low risk of bias when total score was $8-10$, moderate risk of bias when total score was $>5$ and $<8$, and high risk of bias when total score was $>2$ and $\leq 5$. Studies with total score $\leq 2$ were rejected.

\section{Data analysis}

Data for each outcome were analysed using the Comprehensive Meta-Analysis Software, version 3.3 (Biostat, Englewood, NJ, USA). Fixed or random effect meta-analyses were conducted to assess test/placebo differences across studies, with effect size measured as difference in means (DM) and 95\% confidence intervals (CI). In studies with more than one time point, only the longest time point 


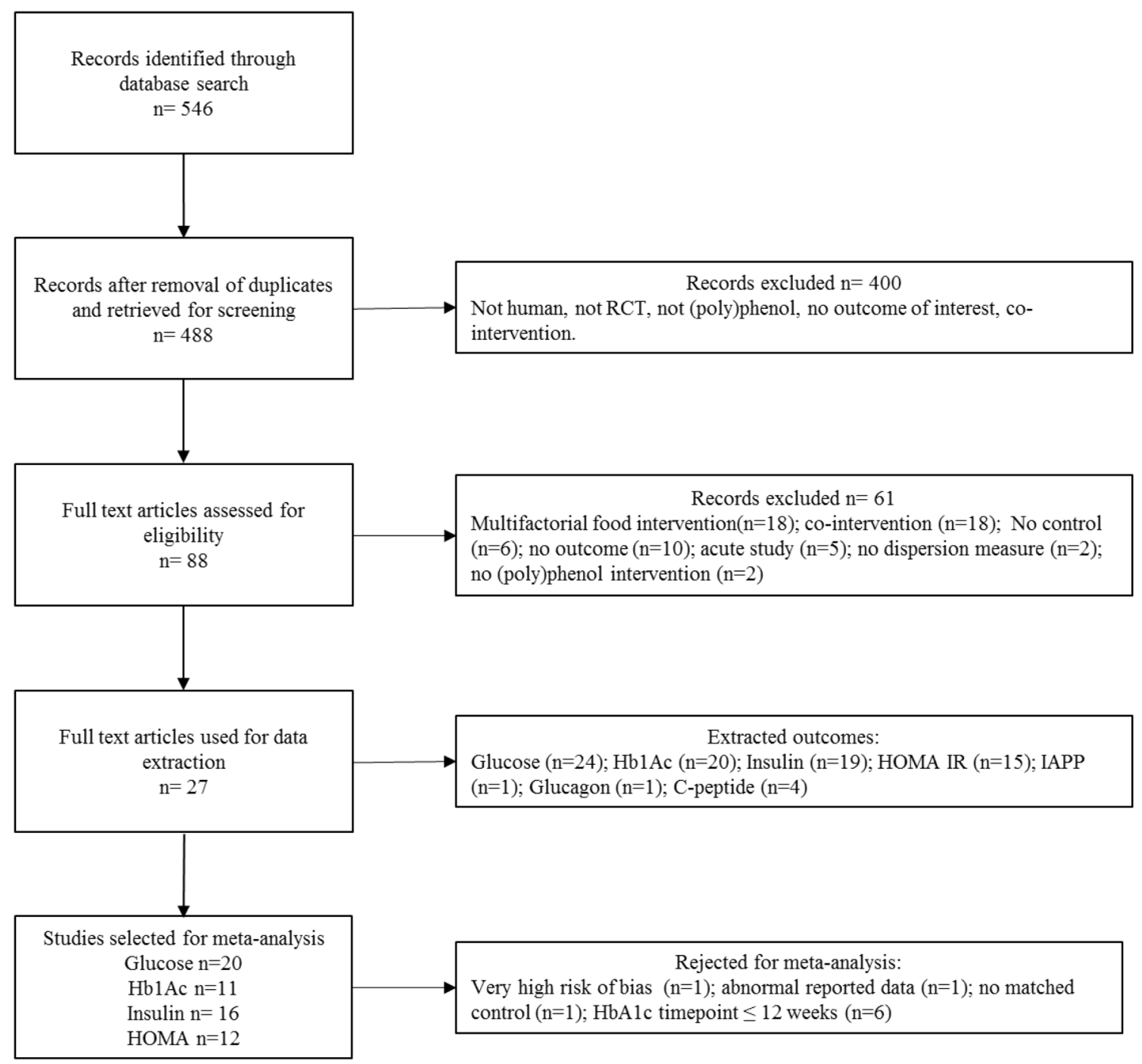

Fig. 1 Flow diagram of the studies selection process for meta-analysis

was included in the analysis, except for the comparative analysis of duration subgroups. Studies with participants receiving different types of supplementation (different doses or types of (poly)phenols) were considered as substudies and the data of sub-studies treated as independent studies. The heterogeneity of studies was assessed using the Cochran's $Q$ statistic (a Chi-squared test with $n-1$ degrees of freedom), and the inconsistency index $I^{2}$ (the proportion of total variation contributed by between-study variability), where $I^{2}$ values equal to $25 \%, 50 \%$ and $75 \%$ were considered as low, moderate and high heterogeneity, respectively [40]. Random effect meta-analysis was used when moderate to high heterogeneity across studies was present and $P$ value for Cochran's $Q$ statistic was lower than 0.1. Publication bias was assessed visually with funnel plots and statistically by applying the Egger's regression test.
Statistical comparisons between subgroups were performed by applying a random-effects analysis and calculation of the between-categories $Q$ statistic and corresponding $P$ values. A $P$ value $<0.05$ was considered statistically significant.

\section{Results}

\section{Description of the included studies}

The study selection process is shown in Fig. 1. A total of 546 studies were retrieved from the electronic reference databases. After initial screening of 488 references, 88 trials met inclusion criteria for full text review. Detailed full text analysis led to exclusion of 61 studies, ending with 27 trials selected for data extraction. Quality analysis pinpointed 
one RCT [41] with a very high risk of bias (score $\leq 2$ ) and thus it was also excluded from the meta-analysis. Only one study referred to IAPP [42] and glucagon [43] and three studies measured C-peptide [43-45]. Neither pro-insulin nor pro-IAPP were further evaluated due to the lack of studies. All RCTs with duration between 4 weeks and 1 year were included for the meta-analysis of glucose (20 RCTs), insulin (16 RCTs) and HOMA-IR (12 RCTs). As Hb1 Ac represents the average level of blood glucose for the past 3 months, only RCTs with duration of 12 weeks or longer were included in the meta-analysis of this biomarker (11 RCTs).

The individuals included in the studies had an average age of 55.5 years, with no predominance of either sex. These individuals presented an average BMI of 28.9, corresponding to overweight. In terms of health status, there were 12 studies with individuals with diagnosed diabetes, 3 studies with individuals classified as at high risk of developing diabetes (participants with metabolic syndrome or impaired glucose tolerance) [46-48], and 5 studies with individuals classified as moderate risk of developing diabetes (overweight, obesity, first-degree relatives with diabetes or peripheral insulin resistance) [43, 49-52]. From the studies including individuals with diabetes the duration of the disease ranges from 2 to 22 years. Individuals from 10 of the 20 studies were undergoing medication for diabetes, antihypertensive drugs, cholesterol-lowering drugs or a combination of these.

Characteristics of the studies included in the meta-analysis are shown in Table 1. Most RCTs were from European countries [43, 44, 46, 47, 49-51, 53-56], seven in Asian countries [45, 48, 52, 57-60], one was performed in North America [61] and one in Australia [62]. The present metaanalysis evaluated the results from RCTs that studied the effect of pure or enriched (poly)phenol extracts intake on individuals with diabetes or at risk of diabetes: six studies used a mixture of (poly)phenols from passion fruit, grape, pine tree bark, among others (doses of 125-2093 mg/day); five studies used isoflavones (doses of 33-100 mg/day); four studies used flavanols (doses of 560-1270 mg/day); one study used anthocyanins (392 mg/day); and five studies used resveratrol (doses of $40-1500 \mathrm{mg} /$ day) (Table 1). ${ }^{1}$

\section{Effect of (poly)phenol supplementation on measures of glucose, $\mathrm{Hb} 1 \mathrm{Ac}$, insulin, and HOMA-IR}

Glucose meta-analysis included 20 RCT studies (two of the RCTs included two sub-studies), with 1200 participants: 681 treated with (poly)phenol supplement and 519 on placebo.

\footnotetext{
1 The total number of studies is equal to 21 because one of the studies depicts results from 2 interventions (a (poly)phenol mixture and resveratrol).
}

Meta-analysis showed a statistically significant reduction in fasting plasma glucose after supplementation with a pure (poly)phenol or enriched extract $(\mathrm{DM}=-3.50 \mathrm{mg} / \mathrm{dL} ; 95 \%$ CI $-6.28,-0.73 ; P=0.013$ ) (Table 2 ). Sensitivity analysis by removal of one study [60] with high risk of bias had no impact on effect size or significance (DM $=-3.32 \mathrm{mg} /$ $\mathrm{dL} ; 95 \% \mathrm{CI}-5.86,-0.77 ; P=0.011$ ) (Fig. 2). There was moderate heterogeneity across the 19 studies with low and moderate risk of bias $\left(I^{2}=47.96 \%\right.$ and $P$ value for $Q$ test $=0.007)$, but no evidence of publication bias $(P$ value for Egger's weighted regression $=0.408$ ).

Meta-analysis on $\mathrm{Hb} 1 \mathrm{Ac}$ with all the 11 studies (including two sub-studies) with 12 or more weeks of intervention (340 participants treated with (poly)phenol and 276 controls) also showed a statistically significant but moderate reduction after supplementation with (poly)phenols ( $\mathrm{DM}=-0.24 \mathrm{mg}$ / $\mathrm{dL}, \mathrm{CI}-0.430,-0.044 ; P=0.016$ ) (Table 2). However, when the high risk of bias study [60] was excluded, significance was lost $(\mathrm{DM}=-0.171 \mathrm{mg} / \mathrm{dL}, \mathrm{CI}-0.409,0.066$; $P=0.158)$ (Fig. 3).

Supplementation with (poly)phenols had no statistically significant effect on insulin and HOMA-IR measures. All studies used for meta-analysis were low and moderate risk of bias and sensitivity analysis by removal of one study had no impact on effect size or $P$ value (Table 2, Figs. 4,5 ). There was low heterogeneity among studies (Table 2 ) and no evidence of publication bias ( $P$ value for Egger's weighted regression was 0.705 for insulin and 0.163 for HOMA-IR).

\section{Subgroup analyses for identification of factors impacting plasma glucose reduction}

To explore the factors that could influence the inter-individual variability of response to (poly)phenol intake, subgroup analyses were performed only on blood glucose. As recommended in Cochrane Handbook for Systematic Reviews of Interventions [38], the study with high risk of bias was excluded from the subgroup analysis [60] to decrease heterogeneity among subgroups and prevent misleading results. The other outcomes were not considered for subgroup analysis due to the low number of studies.

\section{Influence of type of (poly)phenol, dose and duration}

Subgroup analysis on type of (poly)phenol was performed with four subgroups: (poly)phenol mixtures, isoflavones, flavanols, and resveratrol (Table 3). Only one study used anthocyanins as the intervention (poly)phenol [59] and thus, it was excluded from this subgroup analysis. The analysis of the intervention with each separate group of (poly)phenols showed no statistically significant effect. There was no evidence of heterogeneity between subgroups (Table 3 ). 


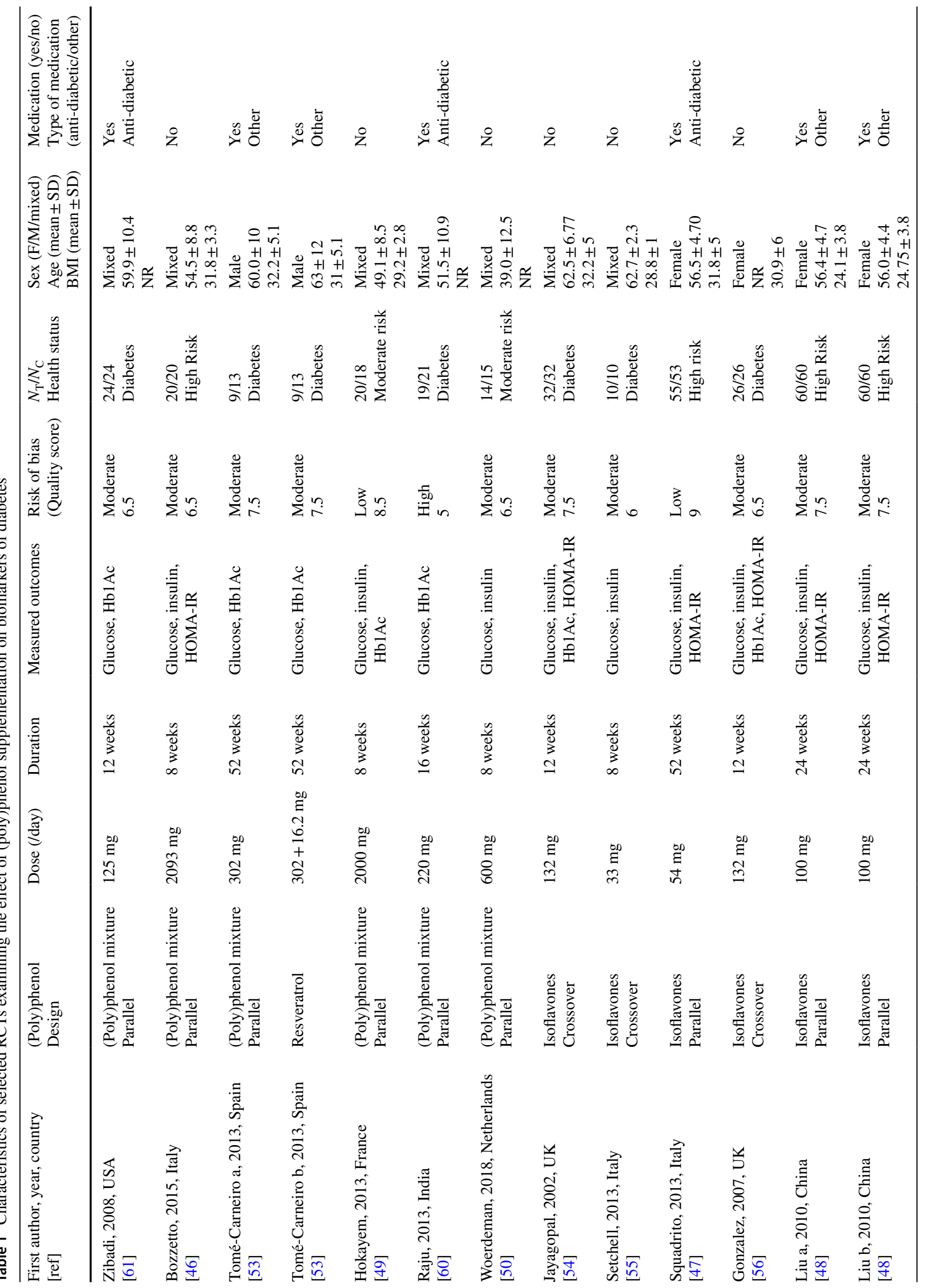




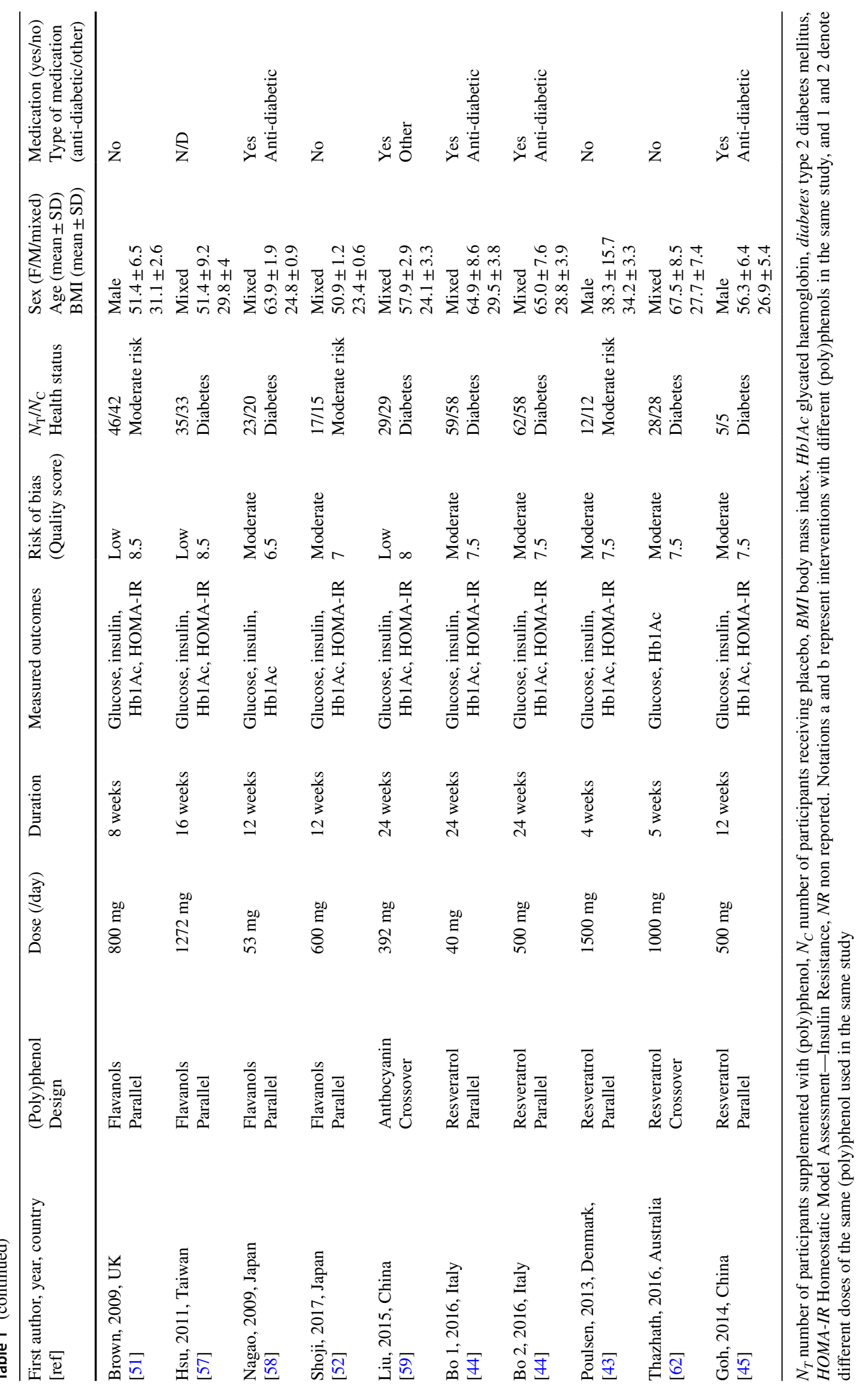


Table 2 Overall effect of (poly) phenol supplementation on measures of glucose, $\mathrm{Hb} 1 \mathrm{Ac}$, insulin and HOMA-IR

\begin{tabular}{|c|c|c|c|c|c|c|c|c|}
\hline & Glucose & & $\mathrm{Hb} 1 \mathrm{Ac}$ & & Insulin & & HOMA-IR & \\
\hline \multicolumn{9}{|l|}{ Effect size } \\
\hline$n\left(N_{\mathrm{T}} / N_{\mathrm{C}}\right)$ & $22(681 / 519)$ & & $13(501 / 429)$ & & $18(601 / / 443)$ & & $14(518 / 351)$ & \\
\hline $\mathrm{DM}$ & $-3.50 \mathrm{mg} / \mathrm{dL}$ & & $-0.24 \%$ & & $0.033 \mu \mathrm{IU} / \mathrm{mL}$ & & 0.030 & \\
\hline $95 \% \mathrm{CI}$ & -6.28 & -0.73 & -0.430 & -0.044 & -0.703 & 0.770 & -0.103 & 0.163 \\
\hline$P$ value & 0.013 & & 0.016 & & 0.929 & & 0.661 & \\
\hline \multicolumn{9}{|c|}{ Heterogeneity } \\
\hline$Q$ & 47.41 & & 33.90 & & 21.63 & & 15.107 & \\
\hline$P$ value & 0.001 & & 0.001 & & 0.199 & & 0.301 & \\
\hline$I^{2}$ & 53.60 & & 64.61 & & 21.41 & & 13.95 & \\
\hline
\end{tabular}

All studies were used in the analysis, including the high risk of bias study. Interventions with different concentrations or different (poly)phenols in the same study were counted as different studies

$n$ number of studies, $N_{T}$ number of participants supplemented with (poly)phenol, $N_{C}$ number of participants receiving placebo, $D M$ difference in means, $C I$ confidence interval, $Q$ Chi-squared statistic, $I^{2}$ inconsistency index, HblAc glycated haemoglobin, HOMA-IR homeostatic model assessment-insulin resistance
For dose subgroups analysis, 19 studies were separated into three subgroups: low dose ( $\geq 100 \mathrm{mg} /$ day); medium dose ( $>100, \geq 500 \mathrm{mg} /$ day); and high dose ( $>500 \mathrm{mg} /$ day) (Table 3). No heterogeneity was evident between dose subgroups. None of the dose subgroups had a statistically significant effect. To assess the impact of study duration, all 32 time points reported across all studies (originated from interventions with different durations and (poly)phenols in the same study) were used for the subgroup analysis. Four subgroups were defined: very short ( $<8$ weeks), short ( $\geq 8,<12$ weeks), medium $(\geq 12,<24$ weeks) and long ( $\geq 24$ weeks). The levels of glucose were statistically significantly reduced only in the medium duration. (Table 3). Nevertheless, this result was not consistent with meta-regression analysis on dose and duration, which showed no significant impact of covariates dose (covariate coefficient $0.002 ; 95 \%$ CI $-0.001 ; 0.006 ; P=0.141$ ) or duration (covariate coefficient $-0.002 ; 95 \% \mathrm{CI}-0.280 ; 0.240 ; P=0.880$ ). Furthermore, the meta-regression analysis showed evidence of variance between studies $\left(\mathrm{Tau}^{2}=15.65 ; I^{2}=52.43 \% ; Q=63.06\right.$; $d f=30 ; P=0.0004)$ from which only $2 \%$ could be explained by dose and duration $\left(R^{2}\right.$ analogue $\left.=0.02\right)$.

\section{Influence of health status of the participants and medication}

Subgroup analysis shows no influence of sex and BMI in the blood glucose effect. As for the analysis of the influence of the health status of the participants, the 19 selected studies were divided into three groups: participants with diabetes, participants at high risk of diabetes and participants at low risk of diabetes. There were 11 studies (including 2 substudies) conducted in participants with diabetes (343 treated with (poly)phenol supplement and 347 controls) [44, 45, 53-59, 61, 62]. Three studies (1 sub-study) included participants at high risk of diabetes: two with metabolic syndrome
[46, 47] (75 treated and 73 controls) and one with impaired glucose tolerance [48] (360 treated and 180 controls). Lastly, five studies were conducted with volunteers considered at moderate risk of diabetes due to: excess weight, obesity, first-degree relatives of individuals with diagnosed diabetes or peripheral insulin resistance [43, 49-52] (109 treated with (poly)phenol supplement and 102 controls). Comparison between the three subgroups showed a statistically significant effect only in the group with diabetes, with a higher blood glucose reduction than the overall effect $(-5.86$ vs $-3.50 \mathrm{mg} / \mathrm{dL}, 95 \% \mathrm{CI}-11.34,-3.89 ; P=0.036)$ (Table 4). No significant heterogeneity between subgroups was observed. Within the diabetes group, when comparing medicated $v s$. non-medicated participants, a higher reduction was observed in the medicated participants $(-6.09 \mathrm{mg} / \mathrm{dL}$, $95 \% \mathrm{CI}-11.35,-0.82 ; P=0.023$ ) (Table 4$)$. This reduction does not seem to be related with differences in average age (57.75 vs. 53.2 years) and BMI (28.4 vs. 29.4) for medicated and non-medicated individuals, respectively. As we observed a more pronounced plasma glucose reduction in medicated participants, we looked for differences between types of medication and divided medicated participants in two subgroups: anti-diabetic medication (biguanides, sulfonylureas, glitazones, glinides and incretins) and other types of medication (statins, antihypertensive, beta-blockers). Data analysis showed a statistically significant glucose reduction in both subgroups but showed a stronger effect in participants with anti-diabetic medication $(-10.17 \mathrm{mg} / \mathrm{dL}, 95 \%$ CI $-16.59,-3.75 ; P=0.002$ ) (Table 4$)$.

Meta regression analysis also pointed to an important impact of health status and medication on the blood glucose level response to (poly)phenol supplementation. A statistically significant between-study variance was observed $\left(\mathrm{Tau}^{2}=15.65 ; I^{2}=52.43 \% ; Q=63.06 ; d f=30 ; P=0.0004\right)$, with $52 \%$ of total between study variance explained by 


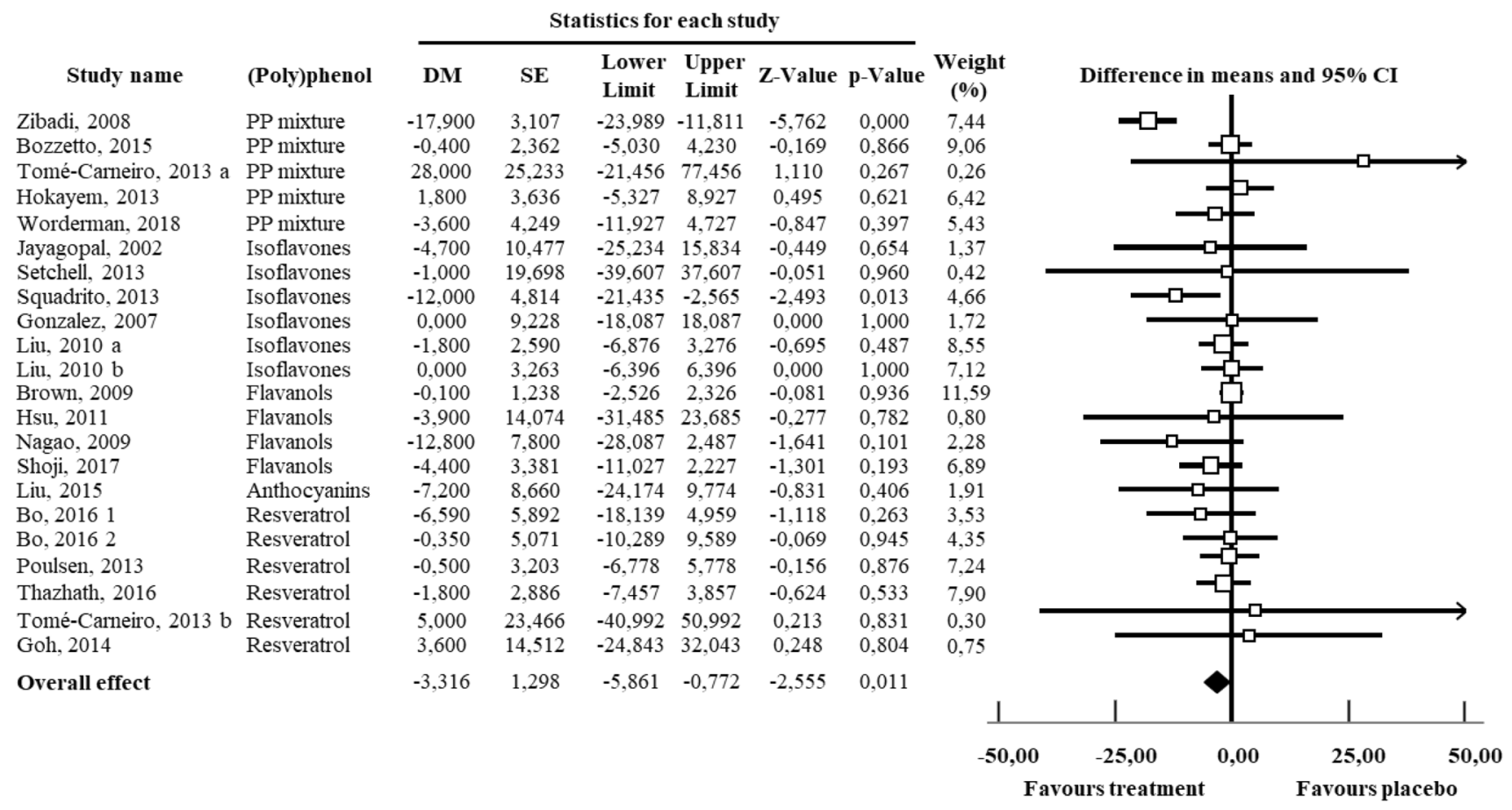

Fig. 2 Effect of (poly)phenol supplementation on plasma glucose $(\mathrm{mg} / \mathrm{dL})$. All studies were used for random effect model meta-analysis. Only low and moderate bias studies are presented. Notations a and $b$ represent interventions with different (poly)phenols in the same study and 1 and 2 denote different doses of the same (poly)phenol used in the same study. $D M$ difference in means, $S E$ standard error, $C I$ confidence interval, $P P$ (poly)phenol

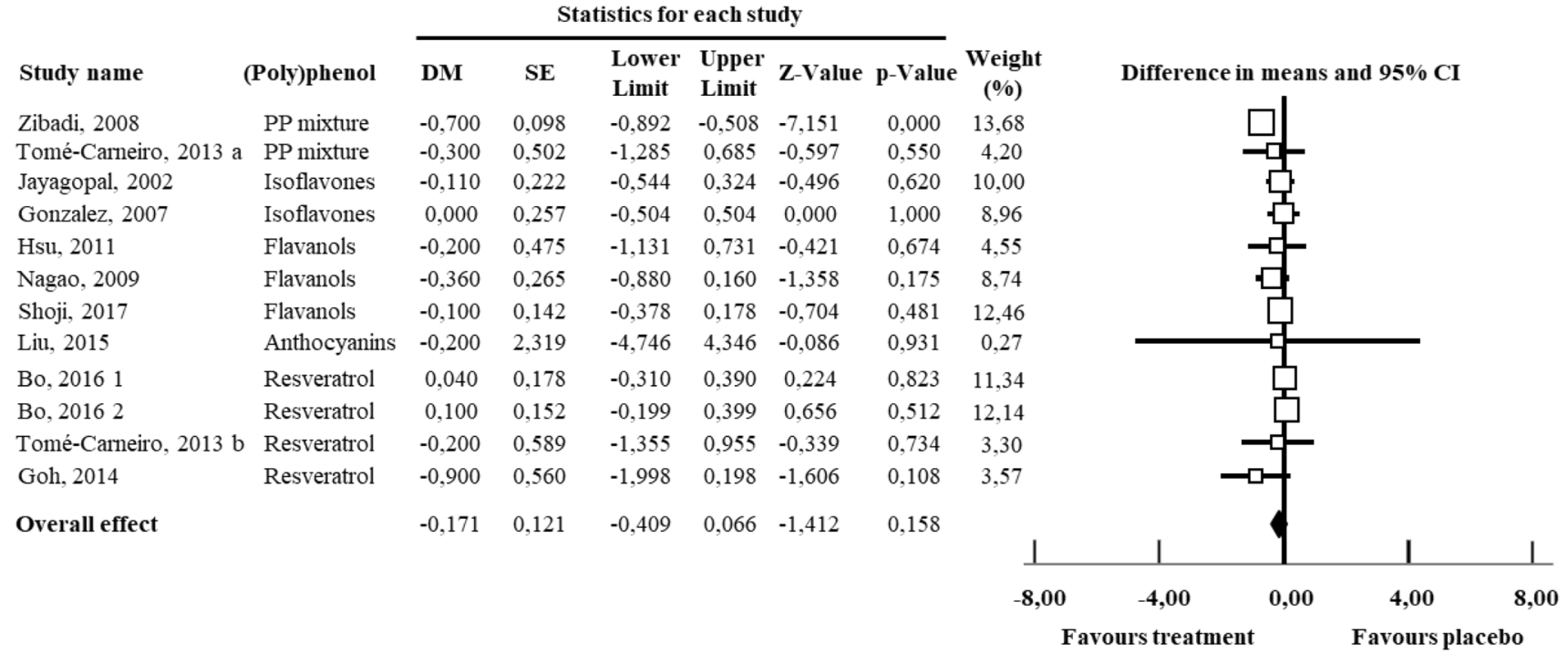

Fig. 3 Effect of (poly)phenol supplementation on glycated haemoglobin (\%). All studies were used for random effect model meta-analysis. Only low and moderate bias studies are presented. Notations a and b represent different times of intervention in the same study and 1 and 2 denote different doses used in the same study. $D M$ difference in means. $S E$ standard error, $C I$ confidence interval, $P P$ (poly)phenol 


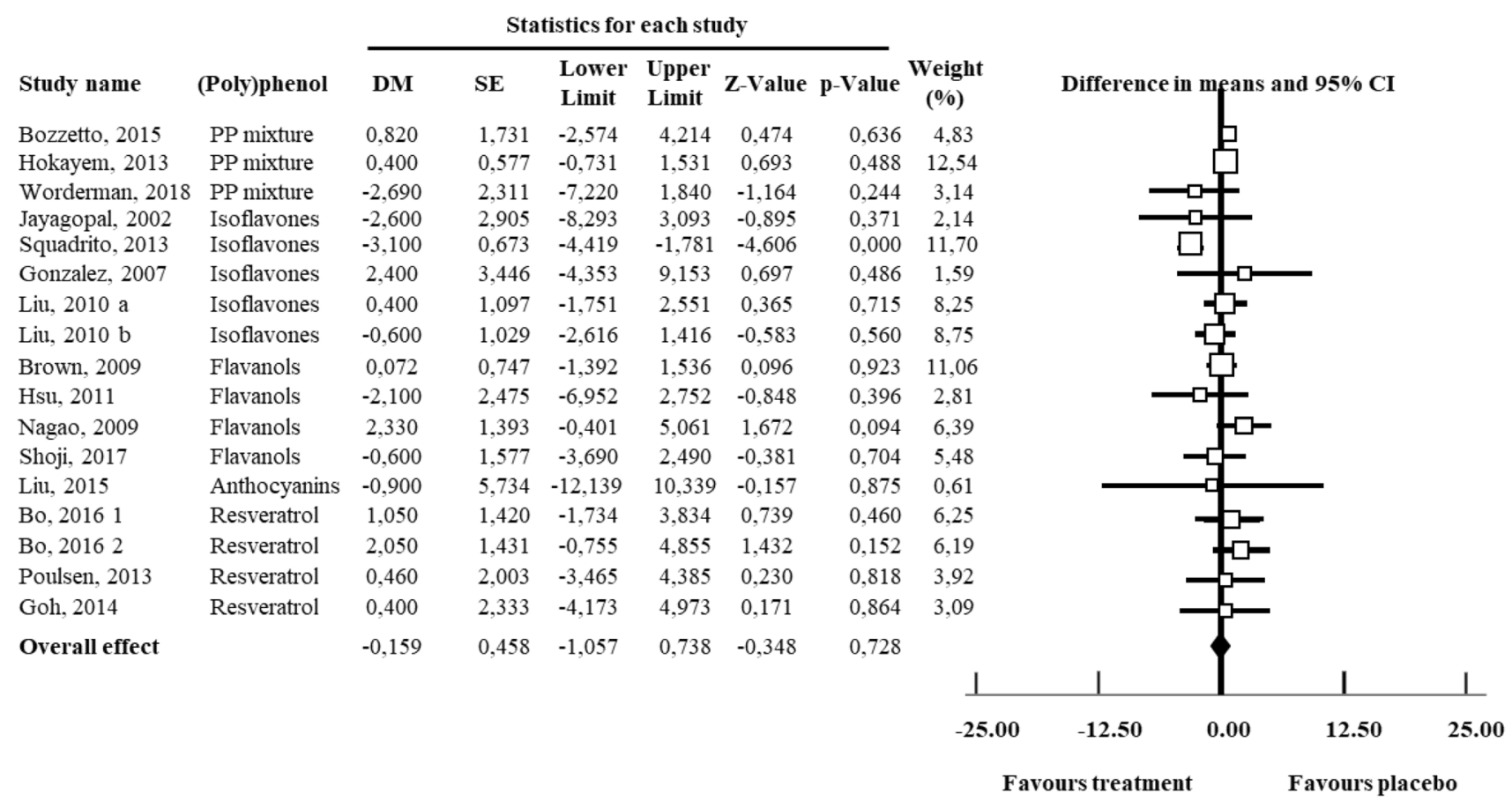

Fig. 4 Effect of (poly)phenol supplementation on insulin $(\mu \mathrm{UI} / \mathrm{mL})$. All studies were used for random effect model meta-analysis. Only low and moderate bias studies are presented. Notations a and b rep- resent different times of intervention in the same study and 1 and 2 denote different doses used in the same study. $D M$ difference in means, $S E$ standard error, $C I$ confidence interval, $P P$ (poly)phenol

Statistics for each study

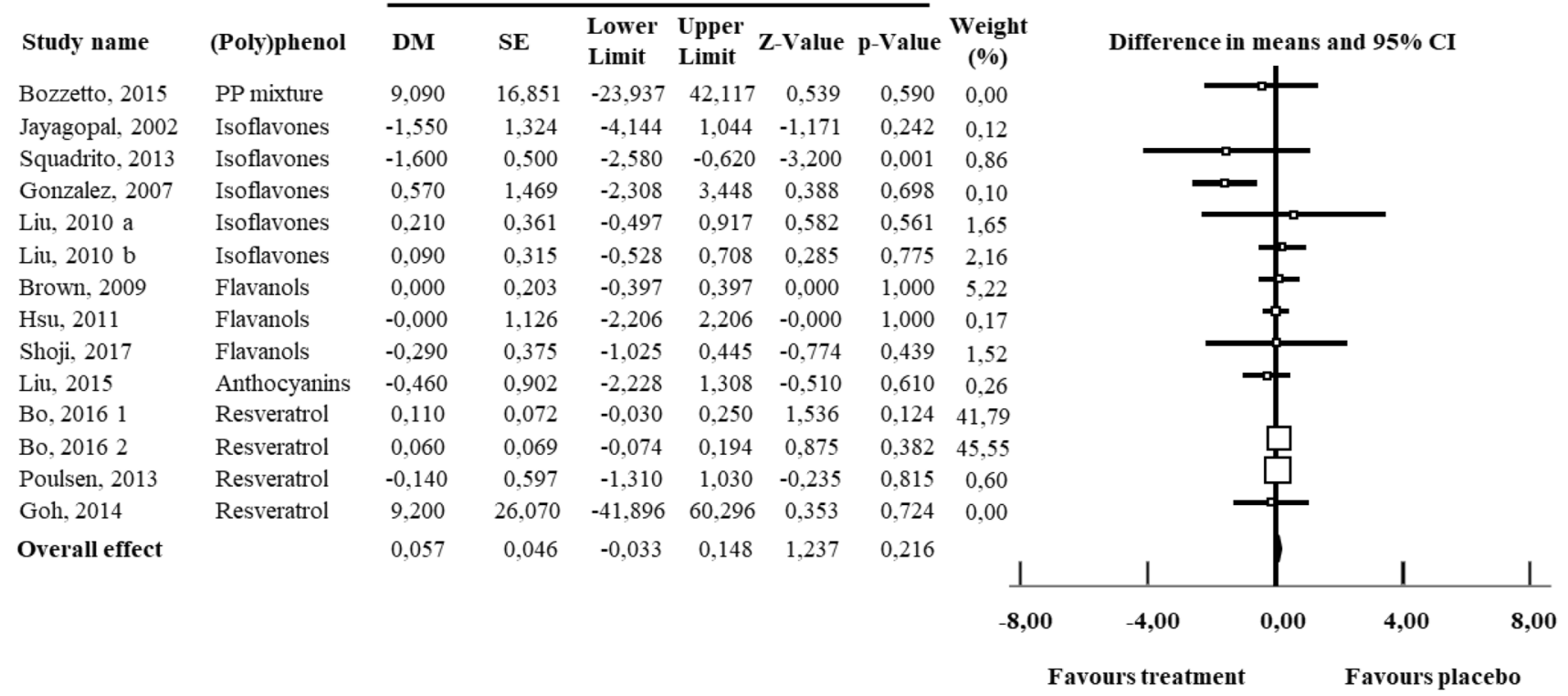

Fig. 5 Effect of (poly)phenol supplementation on HOMA-IR. Only low and moderate bias studies used for fixed effect model meta-analysis. DM difference in means, $S E$ standard error, $C I$ confidence interval, $P P$ (poly)phenol 
Table 3 Subgroup analysis of studies measuring glucose: impact of (poly)phenol type, dose and duration of intervention

\begin{tabular}{|c|c|c|c|c|c|c|}
\hline & \multirow[t]{2}{*}{$n\left(N_{\mathrm{T}} / N_{\mathrm{C}}\right)$} & \multicolumn{4}{|c|}{ Intervention effect } & \multirow{2}{*}{$\begin{array}{l}\text { Heterogeneity between studies } \\
Q ; d f ; P\end{array}$} \\
\hline & & $\mathrm{DM}(\mathrm{mg} / \mathrm{dL})$ & $95 \% \mathrm{CI}$ & & $P$ value & \\
\hline \multicolumn{7}{|l|}{ (Poly)phenol subgroups } \\
\hline (Poly)phenol mixtures & $5(97 / 98)$ & -4.08 & -13.13 & 4.98 & 0.377 & $0.710 ; 4.000 ; 0.950$ \\
\hline Isoflavones & $6(243 / 147)$ & -2.69 & -6.21 & 0.84 & 0.135 & \\
\hline Flavanols & $4(137 / 129)$ & -2.04 & -5.97 & 1.89 & 0.309 & \\
\hline Resveratrol & $6(175 / 116)$ & -1.52 & -5.14 & 2.11 & 0.413 & \\
\hline \multicolumn{7}{|l|}{ Dose subgroups $^{\text {a }}$} \\
\hline High & $8(231 / 222)$ & -1.00 & -2.78 & 0.78 & 0.270 & $2.553 ; 3.000 ; 0.466$ \\
\hline Low & $5(260 / 194)$ & -3.48 & -7.65 & 0.69 & 0.102 & \\
\hline Medium & $8(206 / 208)$ & -5.03 & -13.92 & 3.87 & 0.268 & \\
\hline \multicolumn{7}{|l|}{ Duration $^{\text {b }}$} \\
\hline Long & $9(334 / 179)$ & -4.14 & -8.66 & 0.38 & 0.072 & $1.501 ; 3.000 ; 0.682$ \\
\hline Medium & $11(197 / 195)$ & -6.53 & -12.44 & -0.61 & 0.031 & \\
\hline Short & $7(144 / 139)$ & -2.67 & -6.32 & 0.97 & 0.150 & \\
\hline Very short & $5(40 / 40)$ & -2.77 & -5.76 & 0.22 & 0.069 & \\
\hline
\end{tabular}

Only low and moderate risk of bias studies were used in the analysis. Interventions with different concentrations of or different (poly)phenols in the same work are counted as different studies. Only the last timepoint was used in the analysis except in the Duration subgroup analysis

$n$ number of studies, $N_{T}$ number of participants supplemented with (poly)phenol, $N_{C}$ number of participants receiving placebo, $D M$ difference in means, $C I$ confidence interval, $Q$ Chi-squared statistic, $d f$ degree of freedom.

${ }^{\mathrm{a}}$ Low dose ( $\leq 100 \mathrm{mg} /$ day $)$; medium dose ( $>100, \leq 500 \mathrm{mg} /$ day $)$; and high dose ( $>500 \mathrm{mg} /$ day $)$

${ }^{\mathrm{b}}$ Very short ( $<8$ weeks), short $(\geq 8,<12$ weeks), medium ( $\geq 12,<24$ weeks); long ( $\geq 24$ weeks)

Table 4 Subgroup analysis of studies measuring glucose: impact of health status and medication

\begin{tabular}{lllllll}
\hline & $n$ & DM $(\mathrm{mg} / \mathrm{dL})$ & $95 \% \mathrm{CI}$ & & $P$ value & $Q ; d f ; P$ \\
\hline Health status & & & & & & \\
Diabetes & 13 & -5.86 & -11.34 & -0.39 & 0.036 & $3.403 ; 2.000 ; 0.182$ \\
High risk & 4 & -2.29 & -6.22 & 1.63 & 0.252 & \\
Moderate risk & 5 & -0.58 & -2.57 & 1.41 & 0.568 & \\
Medication & & & & & & \\
Medicated & 12 & -6.09 & -11.35 & -0.82 & 0.023 & $3.642 ; 1.000 ; 0.056$ \\
Non-medicated & 10 & -0.69 & -2.42 & 1.03 & 0.431 & \\
Anti-diabetic med & 6 & -10.17 & -16.59 & -3.75 & 0.002 & \\
Non-anti-diabetic med & 5 & -3.59 & -7.02 & -0.169 & 0.040 & \\
Non defined & 1 & & & & & \\
\hline
\end{tabular}

Only low and moderate risk of bias studies were used in the subgroup analysis. Interventions with different concentrations of or different (poly)phenols in the same work are counted as different studies

Diabetes type 2 diabetes mellitus, $n$ number of studies, $D M$ difference in means, $C I$ confidence interval, $Q$ Chi-squared statistic, $d f$ degree of freedom health status $\left(R^{2}\right.$ analogue $\left.=0.52\right)$, and $34 \%$ explained by medication $\left(R^{2}\right.$ analogue $\left.=0.34\right)$.

\section{Discussion}

This study evaluates and reports the effects of supplementation with various (poly)phenols, whether pure or enriched fractions, excluding interventions using (poly)phenol enriched foods or multifactorial food interventions. The effects on a range of diabetes biomarkers, specifically those most commonly used in clinical practice-blood glucose and $\mathrm{Hb} 1 \mathrm{Ac}$ - and others related with pancreatic function and insulin resistance-insulin and HOMA-IR were evaluated. Overall, the results of this meta-analysis give evidence of a beneficial effect of (poly)phenol supplementation in blood glucose levels of individuals with diabetes and in those at risk of developing the disease.

We also observed a slight reduction in $\mathrm{Hb} 1 \mathrm{Ac}(0.24 \%)$, although statistical significance was lost upon sensitivity 
analysis. In addition, given that an alteration of $30 \mathrm{mg} / \mathrm{dL}$ in blood glucose reflects $1 \%$ change in Hb1Ac marker [63], the $10 \mathrm{mg} / \mathrm{dL}$ reduction in blood glucose levels observed in this meta-analysis is not expected to cause a marked change in Hb1 Ac. Recent meta-analysis encountered an effect on $\mathrm{HbA} 1 \mathrm{c}$ of individuals with diabetes $(-0.21 \pm 0.04)$, but not on individuals without diabetes, subjected to nutritional interventions with $0.028-1.5 \mathrm{~g}$ of extracts, supplements, or foods for $0.7-12$ months [36]. Differences on results may be due to the fact that the authors included interventions with both supplements and foods in their meta-analysis, and we excluded food-based trials from our meta-analysis.

In an attempt to identify some of the factors that may influence the glycaemic response to (poly)phenol intake, we compared the effects of a mixture of (poly)phenols, isoflavones, flavanols and resveratrol on blood glucose. Our analysis shows no statistically significant effects or differences between the four subgroups analysed. Although we cannot discard that these results may be due to the small number of studies per subgroup, it is also plausible that different (poly)phenols may have common and non-specific regulatory effects on the metabolism of glucose. In support of this hypothesis, previous meta-analyses have shown a significant reduction in fasting blood glucose after supplementation with flavonols [39], and a modulation of insulin and HOMA-IR by flavanols present in tea, apple and cocoa [64]. Also, a long-term trial found a correlation between flavan-3-ols and isoflavones intake and the increase in insulin sensitivity [65]. On the other hand, a meta-analysis on the effects of resveratrol has shown a beneficial effect on $\mathrm{Hb} 1 \mathrm{Ac}$ (mean effect size $=0.43$; SE 0.16; 95\% CI $0.10,-0.75 ; P<0.01$ ) but not on blood glucose, insulin and HOMA-IR [66]. Additionally, a recent metaanalysis shows an inverse association between the intake of (poly)phenols and diabetes (HR of 0.56) [35]. These data support the notion that the effector regulators of glucose metabolism at the cellular and organ level may not be the parent compounds supplied to the volunteers. The putative compounds are common low-molecular weight phenolic metabolites of the main classes of (poly)phenols generated in the intestinal tract as the culmination of multiples steps involving gut microbiota metabolism [64, 67-69]. In support of this view, it was recently shown that 2,3-dihydroxybenzoic acid (DHBA), a colonic phenolic acid derived from flavonoid intake, decreases the uptake of glucose and the enzymes responsible for gluconeogenesis in a renal proximal tubular cell line [70], suggesting a possible influence in renal glucose reabsorption and thus, a potential regulatory effect on blood glucose levels.

Of note, our analysis has shown statistical evidence for a higher hyperglycaemia reduction in medicated as compared to non-medicated individuals with diabetes. Remarkably, the strength of evidence on this reduction was higher for those on medication for diabetes, than on individuals taking other types of medication such as statins, antihypertensive and beta-blockers drugs. This is the first meta-analysis that shows evidence for the impact of the type of medication on the effect of (poly)phenols on glycaemic control in individuals with diabetes. Our data are suggestive of a potential synergistic action of (poly)phenol supplementation and medication mainly for diabetes treatment, opening new venues for the exploitation of (poly)phenol-rich diets as co-adjuvants in diabetes management. This notion is supported by previous animal studies showing an interaction between the intake of (poly)phenol-rich herbs and anti-diabetic medication on glucose control and insulin sensitivity [71, 72].

Monitoring of blood glucose levels is the primary clinical criteria for diabetes diagnosis and control. However, other biomarkers such as glucagon, C-peptide, pro-insulin and IAPP/amylin are also useful to monitor diabetes and pancreatic function. However, only a few studies addressed the impact of (poly)phenols on these parameters. The scarcity of studies investigating the potential protective action of (poly) phenols towards these clinical parameters led to their exclusion of the meta-analysis emphasizing the need of further research in the field.

The main strength of this study is the indication that (poly)phenol consumption may improve glycaemic control in individuals with diabetes, particularly those medicated for the disease. Only clinical trial studies were included in the analysis to eliminate confounding factors that may play a role in nutritional interventions, such as limitations of dietary assessment techniques, displacement of other nutrients or difficulties in assessing baseline dietary status [73]. The detected effects on the major clinical symptom of diabetes, the abnormal levels of blood glucose, makes this study relevant to encourage further investigations towards the use of (poly)phenols together with diabetes medication for an improved glycaemic control.

On the other hand, the reduced number of clinical intervention studies included in this meta-analysis as well as the lack of further information reported in those studies constitutes the main weaknesses of our research. This made impossible to perform further sub-groups analyses and to consider additional factors to those herein analysed. This may also represent the main reason as to why we were not able to detect any other significant results apart from those reported for blood glucose. The timeline of the original search also represents a weakness that may have contributed to these limitations. 


\section{Conclusion}

Despite the considerable number of randomized clinical trials that have so far evaluated the health benefits of several (poly)phenols towards biomarkers of diabetes, there is still a clear need for more intervention studies further demonstrating these effects and investigating in depth the factors that influence inter-individual variability in the response to these compounds. The validation of the reduction of blood glucose by these compounds as well as other potential regulatory effects on other biomarkers should be accompanied by mechanistic studies, with a focus on (poly)phenol microbial metabolism for an accurate characterization of their health benefits towards diabetes management and the combined effects with anti-diabetic drugs.

Acknowledgements The present study was conducted within COST Action FA1403-POSITIVe. iNOVA4Health-UID/ Multi/04462/2019, a program financially supported by Fundação para a Ciência e Tecnologia (FCT)/Ministério da Educação e Ciência, through national funds and co-funded by FEDER under the PT2020 Partnership Agreement is acknowledged. Funding from INTERFACE Programme, through the Innovation, Technology and Circular Economy Fund (FITEC), is gratefully acknowledged. UIDP/04748/2020, a program financially supported by FCT is also acknowledged. This study was also supported by FCT via PTDC/BIA-MOL31104/2017 and UID/ Multi/04462/2019-SubProj iNOVA4Health 44 to RM, SFRH/PD/ BD/135504/2018 to AFR, SFRH/BPD/110426/2015 to RR. Sociedade Portuguesa de Diabetologia for the Nuno Castelo-Branco Prize-2016 attributed to RM and RR is also acknowledged.

\section{Compliance with ethical standards}

Conflict of interest The authors declare no conflict of interests.

\section{References}

1. Cavan D, da Rocha Fernandes J, Makaroff L, Ogurtsova K, Webber S (2015) IDF diabetes atlas, Brussels: international diabetes federation, pp 12-19

2. Sladek R, Rocheleau G, Rung J et al (2007) A genome-wide association study identifies novel risk loci for type 2 diabetes. Nature. https://doi.org/10.1038/nature05616

3. Hu FB, Manson JE, Stampfer MJ et al (2002) Diet, lifestyle, and the risk of type 2 diabetes mellitus in women. Obstet Gynecol Surv. https://doi.org/10.1097/00006254-200203000-00018

4. Wing RR, Lang W, Wadden TA et al (2011) Benefits of modest weight loss in improving cardiovascular risk factors in overweight and obese individuals with type 2 diabetes. Diabetes Care. https ://doi.org/10.2337/dc10-2415

5. Kahn SE (2003) The relative contributions of insulin resistance and beta-cell dysfunction to the pathophysiology of Type 2 diabetes. Diabetologia 46:3-19

6. Kahn SE, Hull RL, Utzschneider KM (2006) Mechanisms linking obesity to insulin resistance and type 2 diabetes. Nature 444:840-846

7. Bergman RN, Finegood DT, Kahn SE (2003) The evolution of beta-cell dysfunction and insulin resistance in type 2 diabetes. Eur J Clin Investig. https://doi.org/10.1046/j.1365-2362.32.s3.5.x
8. Westermark P, Andersson A, Westermark GT (2011) Islet amyloid polypeptide, islet amyloid, and diabetes mellitus. Physiol Rev. https://doi.org/10.1152/physrev.00042.2009

9. Leighton E, Sainsbury CA, Jones GC (2017) A practical review of C-peptide testing in diabetes. Diabetes Ther 8:475-487

10. Westermark P, Grimelius L (1973) The pancreatic islet cells in insular amyloidosis in human diabetic and non-diabetic adults. Acta Pathol Microbiol Scand Sect A Pathol. https://doi. org/10.1111/j.1699-0463.1973.tb03538.x

11. Kahn SE, Halban PA (1997) Release of incompletely processed proinsulin is the cause of the disproportionate proinsulinemia of NIDDM. Diabetes 46:1725-1732

12. Paulsson JF, Andersson A, Westermark P, Westermark GT (2006) Intracellular amyloid-like deposits contain unprocessed pro-islet amyloid polypeptide (proIAPP) in beta cells of transgenic mice overexpressing the gene for human IAPP and transplanted human islets. Diabetologia. https://doi.org/10.1007/s00125-006-0206-7

13. Godoy-Matos AF (2014) The role of glucagon on type 2 diabetes at a glance. Diabetol Metab Syndr 6:91

14. Bravo L (2010) Polyphenols: chemistry, dietary sources, metabolism, and nutritional significance. Nutr Rev. https://doi. org/10.1111/j.1753-4887.1998.tb01670.x

15. Pandey KB, Rizvi SI (2009) Plant polyphenols as dietary antioxidants in human health and disease. Oxid Med Cell Longev $2: 270-278$

16. García-Lafuente A, Guillamón E, Villares A et al (2009) Flavonoids as anti-inflammatory agents: implications in cancer and cardiovascular disease. Inflamm Res 58:537-552

17. Aquilano K, Baldelli S, Rotilio G, Ciriolo MR (2008) Role of nitric oxide synthases in Parkinson's disease: a review on the antioxidant and anti-inflammatory activity of polyphenols. Neurochem Res 33:2416-2426

18. Silveira AC, Dias JP, Santos VM et al (2018) The action of polyphenols in diabetes mellitus and Alzheimer's disease: a common agent for overlapping pathologies. Curr Neuropharmacol. https ://doi.org/10.2174/1570159x16666180803162059

19. Harding AH, Wareham NJ, Bingham SA et al (2008) Plasma vitamin $\mathrm{C}$ level, fruit and vegetable consumption, and the risk of new-onset type 2 diabetes mellitus. The European prospective investigation of Cancer-Norfolk prospective study. Arch Intern Med. https://doi.org/10.1001/archinte.168.14.1493

20. Ford ES, Mokdad AH (2001) Fruit and vegetable consumption and diabetes mellitus incidence among U.S. adults. Prev Med (Baltim). https://doi.org/10.1006/pmed.2000.0772

21. Tresserra-Rimbau A, Guasch-Ferré $M$ et al (2015) Intake of total polyphenols and some classes of polyphenols is inversely associated with diabetes in elderly people at high cardiovascular disease risk. J Nutr. https://doi.org/10.3945/jn.115.223610

22. Grosso G, Stepaniak U, Micek A et al (2017) Dietary polyphenols are inversely associated with metabolic syndrome in Polish adults of the HAPIEE study. Eur J Nutr. https://doi.org/10.1007/ s00394-016-1187-z

23. Nguyen CT, Pham NM, Do VV et al (2017) Soyfood and isoflavone intake and risk of type 2 diabetes in Vietnamese adults. Eur J Clin Nutr. https://doi.org/10.1038/ejcn.2017.76

24. Ley SH, Hamdy O, Mohan V, Hu FB (2014) Prevention and management of type 2 diabetes: dietary components and nutritional strategies. Lancet 383:1999-2007

25. Törrönen R, Sarkkinen E, Tapola N et al (2010) Berries modify the postprandial plasma glucose response to sucrose in healthy subjects. Br J Nutr. https://doi.org/10.1017/S00071145099928 68

26. Hosoda K, Wang MF, Liao ML et al (2003) Antihyperglycemic effect of oolong tea in type 2 diabetes. Diabetes Care. https://doi. org/10.2337/diacare.26.6.1714 
27. Luo C, Yang H, Tang C et al (2015) Kaempferol alleviates insulin resistance via hepatic IKK/NF- $\mathrm{KB}$ signal in type 2 diabetic rats. Int Immunopharmacol. https://doi.org/10.1016/j.intimp.2015.07.018

28. Ahad A, Ganai AA, Mujeeb M, Siddiqui WA (2014) Ellagic acid, an NF- $\kappa \mathrm{B}$ inhibitor, ameliorates renal function in experimental diabetic nephropathy. Chem Biol Interact. https://doi. org/10.1016/j.cbi.2014.05.011

29. Radovan D, Opitz N, Winter R (2009) Fluorescence microscopy studies on islet amyloid polypeptide fibrillation at heterogeneous and cellular membrane interfaces and its inhibition by resveratrol. FEBS Lett. https://doi.org/10.1016/j.febslet.2009.03.059

30. Bruno E, Pereira C, Roman KP et al (2013) IAPP aggregation and cellular toxicity are inhibited by $1,2,3,4,6$-penta- $O$-galloyl- $\beta$-dglucose. Amyloid. https://doi.org/10.3109/13506129.2012.762761

31. Huxley R, Lee CMY, Barzi F et al (2009) Coffee, decaffeinated coffee, and tea consumption in relation to incident type 2 diabetes mellitus: a systematic review with meta-analysis. Arch Intern Med 169:2053-2063

32. Liu K, Zhou R, Wang B et al (2013) Effect of green tea on glucose control and insulin sensitivity: a meta-analysis of 17 randomized controlled trials. Am J Clin Nutr. https://doi.org/10.3945/ ajcn.112.052746

33. Zheng XX, Xu YL, Li SH et al (2013) Effects of green tea catechins with or without caffeine on glycemic control in adults: a meta-analysis of randomized controlled trials. Am J Clin Nutr. https://doi.org/10.3945/ajen.111.032573

34. Pham NM, Van DV, Lee AH (2019) Polyphenol-rich foods and risk of gestational diabetes: a systematic review and meta-analysis. Eur J Clin Nutr 73:647-656

35. Rienks J, Barbaresko J, Oluwagbemigun K et al (2018) Polyphenol exposure and risk of type 2 diabetes: dose-response metaanalyses and systematic review of prospective cohort studies. Am J Clin Nutr 108:49-61

36. Palma-Duran SA, Vlassopoulos A, Lean M et al (2017) Nutritional intervention and impact of polyphenol on glycohemoglobin (HbA1c) in non-diabetic and type 2 diabetic subjects: systematic review and meta-analysis. Crit Rev Food Sci Nutr. https://doi. org/10.1080/10408398.2014.973932

37. Liberati A, Altman DG, Tetzlaff J et al (2009) The PRISMA statement for reporting systematic reviews and meta-analyses of studies that evaluate health care interventions: explanation and elaboration. J Clin Epidemiol. https://doi.org/10.1016/j.jclin epi.2009.06.006

38. Higgins JP, Green S (2011) Cochrane handbook for systematic reviews of interventions version 5.1.0 [updated March 2011]. Cochrane Collab. https://doi.org/10.1002/9780470712184.ch4

39. Menezes R, Rodriguez-Mateos A, Kaltsatou A et al (2017) Impact of flavonols on cardiometabolic biomarkers: a meta-analysis of randomized controlled human trials to explore the role of interindividual variability. Nutrients 9:117

40. Haidich AB (2010) Meta-analysis in medical research. Hippokratia 14:29

41. Spadea L, Balestrazzi E (2001) Treatment of vascular retinopathies with Pycnogenol ${ }^{\circledR}$. Phyther Res. https://doi.org/10.1002/ ptr.853

42. Brasnyó P, Molnár GA, Mohás M et al (2011) Resveratrol improves insulin sensitivity, reduces oxidative stress and activates the Akt pathway in type 2 diabetic patients. Br J Nutr. https://doi. org/10.1017/S0007114511000316

43. Poulsen MM, Vestergaard PF, Clasen BF et al (2013) High-dose resveratrol supplementation in obese men an investigator- initiated, randomized, placebo-controlled clinical trial of substrate metabolism, insulin sensitivity, and body composition. Diabetes. https://doi.org/10.2337/db12-0975

44. Bo S, Ponzo V, Ciccone G et al (2016) Six months of resveratrol supplementation has no measurable effect in type 2 diabetic patients. A randomized, double blind, placebo-controlled trial. Pharmacol Res. https://doi.org/10.1016/j.phrs.2016.08.010

45. Goh KP, Lee HY, Lau DP et al (2014) Effects of resveratrol in patients with type 2 diabetes mellitus on skeletal muscle SIRT1 expression and energy expenditure. Int J Sport Nutr Exerc Metab. https://doi.org/10.1123/ijsnem.2013-0045

46. Bozzetto L, Annuzzi G, Pacini G et al (2015) Polyphenol-rich diets improve glucose metabolism in people at high cardiometabolic risk: a controlled randomised intervention trial. Diabetologia. https://doi.org/10.1007/s00125-015-3592-x

47. Squadrito F, Marini H, Bitto A et al (2013) Genistein in the metabolic syndrome: results of a randomized clinical trial. J Clin Endocrinol Metab. https://doi.org/10.1210/jc.2013-1180

48. Liu ZM, Chen YM, Ho SC et al (2010) Effects of soy protein and isoflavones on glycemic control and insulin sensitivity: a 6-mo double-blind, randomized, placebo-controlled trial in postmenopausal Chinese women with prediabetes or untreated early diabetes. Am J Clin Nutr. https://doi.org/10.3945/ajcn.2009.28813

49. Hokayem M, Blond E, Vidal H et al (2013) Grape polyphenols prevent fructose-induced oxidative stress and insulin resistance in first-degree relatives of type 2 diabetic patients. Diabetes Care. https://doi.org/10.2337/dc12-1652

50. Woerdeman J, Del Rio D, Calani L et al (2018) Red wine polyphenols do not improve obesity-associated insulin resistance: a randomized controlled trial. Diabetes Obes Metab. https://doi. org/10.1111/dom.13044

51. Brown AL, Lane J, Coverly J et al (2009) Effects of dietary supplementation with the green tea polyphenol epigallocatechin-3-gallate on insulin resistance and associated metabolic risk factors: randomized controlled trial. Br J Nutr. https://doi.org/10.1017/ S0007114508047727

52. Shoji T, Yamada M, Miura T et al (2017) Chronic administration of apple polyphenols ameliorates hyperglycaemia in highnormal and borderline subjects: a randomised, placebo-controlled trial. Diabetes Res Clin Pract. https://doi.org/10.1016/j.diabr es.2017.03.028

53. Tomé-Carneiro J, Larrosa M, Yáñez-Gascón MJ et al (2013) Oneyear supplementation with a grape extract containing resveratrol modulates inflammatory-related microRNAs and cytokines expression in peripheral blood mononuclear cells of type 2 diabetes and hypertensive patients with coronary artery disease. Pharmacol Res. https://doi.org/10.1016/j.phrs.2013.03.011

54. Jayagopal V, Albertazzi P, Kilpatrick ES et al (2002) Beneficial effects of soy phytoestrogen intake in postmenopausal women with type 2 diabetes. Diabetes Care. https://doi.org/10.2337/diaca re.25.10.1709

55. Setchell KDR, Nardi E, Battezzati PM et al (2013) Novel soy germ pasta enriched in isoflavones ameliorates gastroparesis in type 2 diabetes: a pilot study. Diabetes Care. https://doi.org/10.2337/ dc12-1615

56. Gonźalez S, Jayagopal V, Kilpatrick ES et al (2007) Effects of isoflavone dietary supplementation on cardiovascular risk factors in type 2 diabetes. Diabetes Care. https://doi.org/10.2337/dc06-1814

57. Hsu CH, Liao YL, Lin SC et al (2011) Does supplementation with green tea extract improve insulin resistance in obese type 2 diabetics? A randomized, double-blind, and placebo-controlled clinical trial. Altern Med Rev 16:157-163

58. Nagao T, Meguro S, Hase T et al (2009) A catechin-rich beverage improves obesity and blood glucose control in patients with type 2 diabetes. Obesity. https://doi.org/10.1038/oby.2008.505

59. Liu Y, Xia M, Sun R et al (2015) Purified anthocyanin supplementation reduces dyslipidemia, enhances antioxidant capacity, and prevents insulin resistance in diabetic patients. J Nutr. https://doi. org/10.3945/jn.114.205674

60. Raju NN, Reddy KK, Kumari KK et al (2013) Efficacy of purple passion fruit peel extract in lowering cardiovascular risk factors in 
type 2 diabetic subjects. J Evid Based Complement Altern Med. https://doi.org/10.1177/2156587213475627

61. Zibadi S, Rohdewald PJ, Park D, Watson RR (2008) Reduction of cardiovascular risk factors in subjects with type 2 diabetes by Pycnogenol supplementation. Nutr Res. https://doi.org/10.1016/j. nutres.2008.03.003

62. Thazhath SS, Wu T, Bound MJ et al (2016) Administration of resveratrol for $5 \mathrm{wk}$ has no effect on glucagon-like peptide 1 secretion, gastric emptying, or glycemic control in type 2 diabetes: a randomized controlled trial. Am J Clin Nutr. https://doi. org/10.3945/ajcn.115.117440

63. Nathan DM, Kuenen J, Borg R et al (2008) Translating the A1C assay into estimated average glucose values. Diabetes Care. https ://doi.org/10.2337/dc08-0545

64. Alshammari N, Palma-Duran SA, Jiang G et al (2018) A systematic review and meta-analysis of randomized controlled trials exploring the role of inter-individual variability on the effect of flavanols on insulin and HOMA-IR. Proc Nutr Soc 77:E40. https ://doi.org/10.1017/S0029665118000344

65. Curtis PJ, Dhatariya K, Sampson M et al (2012) Chronic ingestion of flavan-3-ols and isoflavones improves insulin sensitivity and lipoprotein status and attenuates estimated 10-year CVD risk in medicated postmenopausal women with type 2 diabetes: a 1-year, double-blind, randomized, controlled trial. Diabetes Care. https:// doi.org/10.2337/dc11-1443

66. Hausenblas HA, Schoulda JA, Smoliga JM (2015) Resveratrol treatment as an adjunct to pharmacological management in type 2 diabetes mellitus-systematic review and meta-analysis. Mol Nutr Food Res 59:147-159
67. Pimpão RC, Dew T, Figueira ME et al (2014) Urinary metabolite profiling identifies novel colonic metabolites and conjugates of phenolics in healthy volunteers. Mol Nutr Food Res. https://doi. org/10.1002/mnfr.201300822

68. Williamson G, Clifford MN (2017) Role of the small intestine, colon and microbiota in determining the metabolic fate of polyphenols. Biochem Pharmacol 139:24-39

69. Carregosa D, Carecho RM, Figueira I, Santos CN (2019) Low molecular weight metabolites from polyphenols as effectors for attenuating neuroinflammation. J Agric Food Chem. https://doi. org/10.1021/acs.jafc.9b02155

70. Álvarez-Cilleros D, Martín MÁ, Ramos S (2018) (-)-Epicatechin and the colonic 2,3-dihydroxybenzoic acid metabolite regulate glucose uptake, glucose production, and improve insulin signaling in renal NRK-52E cells. Mol Nutr Food Res. https://doi. org/10.1002/mnfr.201700470

71. Gupta RC, Chang D, Nammi S et al (2017) Interactions between antidiabetic drugs and herbs: an overview of mechanisms of action and clinical implications. Diabetol Metab Syndr 9:59

72. Haritha C, Gopala Reddy A, Ramana Reddy Y, Anilkumar B (2015) Pharmacodynamic interaction of fenugreek, insulin and glimepiride on sero-biochemical parameters in diabetic Sprague-Dawley rats. Vet World. https://doi.org/10.14202/vetwo rld.2015.656-663

73. Weaver CM, Miller JW (2017) Challenges in conducting clinical nutrition research. Nutr Rev. https://doi.org/10.1093/nutrit/nux02 6

\section{Affiliations}

\section{Ana F. Raimundo ${ }^{1,2,3} \cdot$ Filipa Félix $^{1,3} \cdot$ Rita Andrade $^{4} \cdot$ María-Teresa García-Conesa $^{5}$. Antonio González-Sarrías ${ }^{5}$. João Gilsa-Lopes ${ }^{2}$. Dulce do $0^{4}$ - Ana Raimundo ${ }^{4}$. Rogério Ribeiro ${ }^{2,4,6}$. Ana Rodriguez-Mateos ${ }^{7}$. Cláudia N. Santos ${ }^{1,2,3} \cdot$ Manuel Schär $^{8} \cdot$ Ana Silva $^{9} \cdot$ Inês Cruz ${ }^{9} \cdot$ Brian Wang $^{7} \cdot$ Paula Pinto $^{3,9,10} \cdot$ Regina Menezes $^{1,2,3}$ (1)}

1 iBET, Instituto de Biologia Experimental e Tecnológica, Apartado 12, 2781-901 Oeiras, Portugal

2 CEDOC, Chronic Diseases Research Centre, NOVA Medical School, Faculdade de Ciências Médicas, Universidade NOVA de Lisboa, Campo dos Mártires da Pátria, 130, 1169-056 Lisbon, Portugal

3 Instituto de Tecnologia Química e Biológica António Xavier, Universidade Nova de Lisboa, Av. da República, 2780-157 Oeiras, Portugal

4 APDP, Associação Protectora Dos Diabéticos de Portugal, Lisbon, Portugal

5 Research Group On Quality, Safety and Bioactivity of Plant Foods, Department of Food Science and Technology, CEBAS-CSIC, Murcia, Spain

6 iBiMed-UA, Aveiro, Portugal
7 Department of Nutritional Sciences, School of Life Course Sciences, Faculty of Life Sciences and Medicine, King's College London, London, UK

8 Department of Food and Nutritional Sciences, School of Chemistry, Food and Pharmacy, University of Reading, Reading, UK

9 Instituto Politécnico de Santarém, Escola Superior Agrária, S. Pedro, 2001-904 Santarém, Portugal

10 Life Quality Research Centre, Avenida Dr. Mário Soares N. ${ }^{\circ}$ 110, 2040-413 Rio Maior, Portugal 